\title{
Remnant Molecular Clouds in the Ori OB 1 Association
}

\author{
Katsuo Ogura ${ }^{1}$ and Koji Sugitani ${ }^{2}$ \\ ${ }^{1}$ Kokugakuin University, Higashi, Shibuya-ku, Tokyo 150-8440, Japan \\ ogura@kokugakuin.ac.jp \\ ${ }^{2}$ Institute for Natural Sciences, Nagoya-City University, Mizuho-ku, Nagoya 467-8501, Japan \\ Received 1997 August 1, accepted 1998 January 5
}

\begin{abstract}
We suggest and give some evidence that as an HII region expands and the $\mathrm{O}$ stars evolve into $\mathrm{B}$ giants, remains of the molecular clouds first appear as bright-rimmed clouds, then as cometary globules and finally as small clouds which are visible by the reflected light from the $\mathrm{B}$ giants. We propose to call the last of these 'reflection clouds' and all three categories collectively 'remnant clouds'. A list is presented of about 80 objects of these remnant clouds in the Ori OB 1 association. In the Belt region there is a beautiful spatial sequence from bright-rimmed clouds through cometary globules to reflection clouds. We suspect that retarded star formation in remnant clouds can explain the presence of so-called dispersed $\mathrm{T}$ Tau stars in the peripheries of $\mathrm{OB}$ associations.
\end{abstract}

Keywords: ISM: molecular clouds — ISM: globules - HII regions — OB associations stars: formation

\section{Introduction}

It is widely accepted that once OB stars form in a molecular cloud, they disperse it by ionisation or energetic stellar winds. But giant molecular clouds are usually very clumpy (e.g. Tatematsu et al. 1991) and so some of the high-density cores/clumps can persist. They are often found as bright-rimmed clouds or cometary globules in or around HII regions. Bright-rimmed clouds are surrounded and compressed by a curved ionisation/shock front on at least one side of their surface. Cometary globules are also surrounded by an ionisation front, but are found in very extended HII regions like the Gum Nebula or may not be directly associated with an HII region (see the list by Zealey et al. 1983). Therefore they probably correspond to a stage after bright-rimmed clouds. Due to their enhanced gas density, which hydrodynamical simulations of radiation-driven implosion indicate (e.g. Lefloch \& Lazareff 1994), retarded star formation takes place in both bright-rimmed clouds (Sugitani, Fukui \& Ogura 1991; Sugitani \& Ogura 1994; Sugitani, Tamura \& Ogura 1995) and cometary globules (Reipurth 1983).

After OB stars evolve into B giants and stop radiating strong Lyman continuum, cometary globules lose their ionised envelope and so are no longer prominent. Still they could be optically visible by reflecting interstellar light in the OB association, although presumably with a reduced brightness compared, in particular, to bright rims. In an attempt to detect such clouds and examine the above scenario we have undertaken a survey of Schmidt atlases and plates in the Orion OB 1 association and have found many likely objects. We propose calling these 'reflection clouds' and the three categories collectively 'remnant clouds'. Our follow-up observations for some of them confirm their molecular nature and show convincing evidence for low-mass star formation in at least some of them.

\section{Selection Criteria and the Catalogue}

Surveys of the Ori OB 1 Association have been made in a search for candidate reflection clouds as well as bright-rimmed clouds and cometary globules, using the SERC Equatorial EJ Sky Atlas in the b_J band, the Second Palomar Sky Survey B Atlas and deep red plates taken with the UK Schmidt telescope. The survey area is the square of $\mathrm{RA}=4^{\mathrm{h}} 50^{\mathrm{m}}$ to $6^{\mathrm{h}} 10^{\mathrm{m}}$ and $\mathrm{Dec}=-12^{\circ} 30^{\prime}$ to $+7^{\circ} 30^{\prime}$ (1950). The selection criteria are as follows. Bright-rimmed clouds should be immersed in bright HII regions, but contrary to our previous catalogues (Sugitani et al. 1991; Sugitani \& Ogura 1994) we list all of them irrespective of association with IRAS point sources. Cometary globules are located far from bright HII regions or in their peripheries where at most faint $\mathrm{H} \alpha$ background emission is discernible, and their outline should be brighter on the red plates than on the IIIaJ plates, especially at their apex. Reflection clouds should also be located outside bright HII regions, but should be brighter on the IIIaJ plates than on the red plates. Further, they should lack obvious internal illuminating stars and have more or less sharp boundaries in contrast to ordinary reflection nebulae, which are generally very diffuse. 
Table 1. Remnant clouds in the Ori OB 1 association

\begin{tabular}{|c|c|c|c|c|c|}
\hline No. & $\begin{array}{l}\text { RA (1950) } \\
\text { (h:m:s) }\end{array}$ & $\begin{array}{c}\text { Dec }(1950) \\
\left({ }^{\circ}:^{\prime}:^{\prime \prime}\right)\end{array}$ & $\begin{array}{l}\text { Width } \times \text { length } \\
\quad(\operatorname{arcmin})\end{array}$ & Type & Identifications* $^{*}$ \\
\hline 1 & 5:01:57 & $-06: 16: 20$ & $1 \cdot 4 \times 5 \cdot 1$ & $\mathrm{r}$ & \\
\hline 2 & 5:03:48 & $-04: 00: 20$ & $5 \cdot 4 \times 7 \cdot 8$ & $\mathrm{r}$ & CB28 \\
\hline 3 & $5: 04: 25$ & $-03: 24: 10$ & $14 \cdot 0 \times 33 \cdot 6$ & $r ?$ & NGC1788, Mำ13, LBN916, DG51 \\
\hline 4 & $5: 09: 32$ & $-01: 59: 10$ & $3 \cdot 4 \times 7 \cdot 8$ & $\mathrm{r}$ & \\
\hline 5 & $5: 10: 56$ & 06:44:00 & $2 \cdot 1 \times 4 \cdot 5$ & $\mathrm{r}$ & \\
\hline 6 & $5: 14: 36$ & $-07: 47: 50$ & $2 \cdot 5 \times 26 \cdot 9$ & $\mathrm{r}$ & \\
\hline 7 & $5: 17: 13$ & $-05: 54: 20$ & $11 \cdot 2 \times 21 \cdot 3$ & $\mathrm{c}$ & BRC16, $\mathrm{M}^{3} \mathrm{~T} 14, \mathrm{LBN} 960$ \\
\hline 8 & $5: 17: 39$ & $-04: 58: 20$ & $1 \cdot 8 \times 5 \cdot 6$ & $\mathrm{r} ?$ & \\
\hline 9 & $5: 18: 13$ & $-05: 26: 40$ & $5 \cdot 8 \times 8 \cdot 4$ & $\mathrm{r} ?$ & \\
\hline 10 & $5: 18: 21$ & $-05: 52: 40$ & $13 \cdot 4 \times 16 \cdot 8$ & $\mathrm{c}$ & S278, LBN956? \\
\hline 11 & $5: 18: 23$ & $-05: 42: 40$ & $4 \cdot 7 \times 10 \cdot 1$ & $\mathrm{r}$ & \\
\hline 12 & $5: 19: 38$ & $-03: 44: 20$ & $6 \cdot 3 \times 9 \cdot 5$ & c & CB29 \\
\hline 13 & $5: 23: 40$ & $-10: 25: 40$ & $1 \cdot 1 \times 7 \cdot 8$ & $\mathrm{r}$ & \\
\hline 14 & $5: 23: 46$ & $-10: 34: 10$ & $1 \cdot 2 \times 2 \cdot 7$ & $\mathrm{r}$ & \\
\hline 15 & $5: 23: 57$ & $-10: 27: 20$ & $0 \cdot 8 \times 3 \cdot 4$ & $\mathrm{r}$ & \\
\hline 16 & $5: 24: 32$ & $-10: 17: 00$ & $1 \cdot 2 \times 3 \cdot 5$ & $\mathrm{r}$ & \\
\hline $17 \mathrm{~A}$ & $5: 24: 51$ & $-10: 31: 10$ & $2 \cdot 1 \times 3 \cdot 7$ & $\mathrm{r}$ & \\
\hline 17B & $5: 24: 39$ & $-10: 30: 40$ & $0 \cdot 7 \times 1 \cdot 7$ & $\mathrm{r}$ & \\
\hline 18 & $5: 25: 06$ & $-10: 37: 50$ & $0 \cdot 4 \times 1 \cdot 1$ & $\mathrm{r}$ & \\
\hline 19 & $5: 25: 28$ & $-10: 38: 00$ & $1 \cdot 1 \times 2 \cdot 9$ & $\mathrm{r}$ & \\
\hline 20 & $5: 27: 53$ & 03:14:50 & $1 \cdot 0 \times 3 \cdot 4$ & $\mathrm{r}$ & \\
\hline 21 & 5:29:05 & $-02: 15: 00$ & $2 \cdot 3 \times 3 \cdot 9$ & $\mathrm{r} ?$ & \\
\hline 22 & $5: 29: 22$ & $-10: 20: 00$ & $1 \cdot 7 \times 2 \cdot 2$ & $\mathrm{r}$ & \\
\hline 23 & $5: 29: 55$ & $-10: 20: 20$ & $2 \cdot 2 \times 6 \cdot 2$ & $\mathrm{r}$ & \\
\hline 24 & 5:30:00 & $-02: 12: 10$ & $0 \cdot 4 \times 0 \cdot 9$ & $\mathrm{r}$ & \\
\hline $25 \mathrm{~A}$ & $5: 30: 13$ & $-02: 57: 50$ & $7 \cdot 8 \times 8 \cdot 4$ & c & \\
\hline $25 \mathrm{~B}$ & $5: 29: 59$ & $-03: 02: 20$ & $2 \cdot 9 \times 2 \cdot 5$ & $\mathrm{c}$ & \\
\hline 26 & 5:30:17 & $-00: 50: 40$ & $5 \cdot 0 \times 6 \cdot 2$ & $\mathrm{r}$ & \\
\hline 27 & 5:30:51 & $-00: 40: 00$ & $2 \cdot 8 \times 5 \cdot 3$ & $\mathrm{r}$ & IC423, CB31, M ${ }^{3} \mathrm{~T} 28 ?$, LBN913, DG58 \\
\hline 28 & 5:31:05 & $-00: 26: 50$ & $1 \cdot 3 \times 2 \cdot 1$ & $\mathrm{r}$ & IC424, M ${ }^{3} \mathrm{~T} 29 ?$, DG59? \\
\hline $29 \mathrm{~A}$ & $5: 30: 27$ & $-01: 39: 00$ & $0 \cdot 4 \times 0 \cdot 6$ & $\mathrm{r}$ & \\
\hline 29B & 5:30:42 & $-01: 35: 30$ & $1 \cdot 1 \times 2 \cdot 2$ & $\mathrm{r}$ & \\
\hline $29 \mathrm{C}$ & $5: 30: 52$ & $-01: 29: 10$ & $1 \cdot 6 \times 2 \cdot 0$ & $\mathrm{r}$ & \\
\hline 29D & 5:31:00 & $-01: 38: 20$ & $5 \cdot 4 \times 5 \cdot 4$ & c & \\
\hline $29 \mathrm{E}$ & 5:31:05 & $-01: 27: 50$ & $1 \cdot 0 \times 1 \cdot 9$ & $\mathrm{r}$ & \\
\hline $29 \mathrm{~F}$ & 5:31:16 & $-01: 31: 10$ & $1 \cdot 2 \times 1 \cdot 8$ & $\mathrm{r} ?$ & \\
\hline $29 \mathrm{G}$ & $5: 31: 18$ & $-01: 26: 40$ & $2 \cdot 0 \times 3 \cdot 0$ & $\mathrm{r}$ & \\
\hline $29 \mathrm{H}$ & 5:31:39 & $-01: 22: 40$ & $1 \cdot 0 \times 1 \cdot 1$ & $\mathrm{r}$ & \\
\hline 29I & 5:31:44 & $-01: 13: 10$ & $1 \cdot 0 \times 3 \cdot 9$ & $\mathrm{r}$ & LBN935? \\
\hline 29J & $5: 31: 51$ & $-01: 23: 50$ & $0 \cdot 9 \times 2 \cdot 2$ & $\mathrm{r} ?$ & \\
\hline $29 \mathrm{~K}$ & 5:32:08 & $-01: 23: 50$ & $2 \cdot 7 \times 4 \cdot 5$ & $\mathrm{c} ?$ & \\
\hline 29L & 5:32:30 & $-01: 17: 40$ & $2 \cdot 0 \times 3 \cdot 4$ & $\mathrm{r}$ & \\
\hline 30 & $5: 31: 28$ & $-03: 43: 40$ & $5 \cdot 0 \times 5 \cdot 3$ & $c ?$ & $\mathrm{M}^{3} \mathrm{~T} 19 ?$ \\
\hline 31 & $5: 31: 58$ & $-03: 00: 30$ & $3 \cdot 7 \times 7 \cdot 3$ & $\mathrm{c}$ & BRC19 \\
\hline 32 & $5: 32: 25$ & $-00: 35: 50$ & $1 \cdot 0 \times 1 \cdot 3$ & $\mathrm{r}$ & \\
\hline $33 \mathrm{~A}$ & $5: 33: 27$ & $-03: 26: 20$ & $3 \cdot 4 \times 4 \cdot 5$ & $\mathrm{r}$ & \\
\hline 33B & $5: 33: 27$ & $-03: 32: 20$ & $1 \cdot 9 \times 3 \cdot 6$ & $\mathrm{r}$ & \\
\hline 34 & 5:33:44 & $-04: 02: 30$ & $1 \cdot 3 \times 1 \cdot 3$ & $c ?$ & \\
\hline $35 \mathrm{~A}$ & 5:33:56 & $-00: 19: 30$ & $5 \cdot 6 \times 3 \cdot 9$ & $\mathrm{r}$ & IC426, CB32, LBN921, DG61 \\
\hline 35B & 5:34:06 & $-00: 21: 50$ & $1 \cdot 8 \times 3 \cdot 2$ & $\mathrm{r}$ & \\
\hline 36 & $5: 34: 06$ & $-04: 03: 00$ & $2 \cdot 9 \times 2 \cdot 7$ & $c ?$ & \\
\hline 37 & $5: 34: 14$ & $-00: 25: 50$ & $0 \cdot 9 \times 1 \cdot 6$ & $\mathrm{r}$ & \\
\hline 38 & $5: 34: 24$ & $-00: 23: 30$ & $0 \cdot 9 \times 2 \cdot 8$ & $\mathrm{r}$ & DG62 \\
\hline 39 & $5: 34: 40$ & $-00: 22: 50$ & $1 \cdot 1 \times 0 \cdot 9$ & $\mathrm{r}$ & DG63 \\
\hline $40 \mathrm{~A}$ & $5: 35: 33$ & $-01: 46: 40$ & $2 \cdot 8 \times 4 \cdot 5$ & c & Ori I-2, BRC20 \\
\hline $40 \mathrm{~B}$ & $5: 35: 22$ & $-01: 39: 00$ & $0 \cdot 7 \times 1 \cdot 3$ & c & \\
\hline $40 \mathrm{C}$ & 5:35:19 & $-01: 37: 20$ & $0 \cdot 9 \times 1 \cdot 1$ & c & \\
\hline $40 \mathrm{D}$ & $5: 35: 20$ & $-01: 34: 30$ & $1 \cdot 2 \times 2 \cdot 3$ & $\mathrm{c}$ & \\
\hline 41 & 5:35:54 & $-05: 15: 30$ & $2 \cdot 6 \times 8 \cdot 4$ & c & BRC22 \\
\hline 42 & $5: 36: 26$ & $-07: 47: 10$ & $1 \cdot 5 \times 3 \cdot 6$ & c & \\
\hline 43 & $5: 36: 51$ & $-00: 37: 40$ & $1 \cdot 1 \times 2 \cdot 0$ & $\mathrm{r}$ & DG71 \\
\hline $44 \mathrm{~A}$ & $5: 37: 11$ & $-03: 39: 50$ & $4 \cdot 7 \times 4 \cdot 5$ & $\mathrm{~b}$ & BRC21, LBN965 \\
\hline $44 \mathrm{~B}$ & $5: 37: 12$ & $-03: 43: 20$ & $0 \cdot 9 \times 0 \cdot 9$ & $\mathrm{~b}$ & \\
\hline 45 & $5: 37: 15$ & $-05: 22: 40$ & $1 \cdot 9 \times 2 \cdot 5$ & c & \\
\hline 46 & $5: 37: 51$ & $-05: 26: 00$ & $2 \cdot 3 \times 3 \cdot 4$ & $\mathrm{c}$ & \\
\hline
\end{tabular}


Table 1 (Continued)

\begin{tabular}{|c|c|c|c|c|c|}
\hline No. & $\begin{array}{l}\text { RA (1950) } \\
\text { (h:m:s) }\end{array}$ & $\begin{array}{c}\text { Dec }(1950) \\
\left({ }^{\circ}:^{\prime}:^{\prime \prime}\right)\end{array}$ & $\begin{array}{l}\text { Width } \times \text { length } \\
\quad(\text { arcmin) }\end{array}$ & Туре & Identifications* \\
\hline $47 \mathrm{~A}$ & $5: 38: 09$ & $-05: 26: 30$ & $2 \cdot 5 \times 4 \cdot 5$ & c & \\
\hline $47 \mathrm{~B}$ & $5: 38: 31$ & $-05: 28: 10$ & $6 \cdot 2 \times 7 \cdot 3$ & $\mathrm{c}$ & \\
\hline 48 & $5: 37: 56$ & $-00: 31: 30$ & $1 \cdot 2 \times 1 \cdot 8$ & $\mathrm{r}$ & LBN927, DG73 \\
\hline 49 & $5: 38: 17$ & $-02: 14: 10$ & $0 \cdot 7 \times 1 \cdot 0$ & $\mathrm{~b}$ & \\
\hline 50 & $5: 38: 24$ & $-02: 19: 20$ & $1 \cdot 2 \times 1 \cdot 3$ & b & \\
\hline 51 & $5: 38: 26$ & $-02: 11: 50$ & $0 \cdot 6 \times 1 \cdot 2$ & b & \\
\hline 52 & 5:38:30 & $-02: 28: 40$ & $4 \cdot 0 \times 3 \cdot 9$ & b & Horsehead Neb, $\mathrm{M}^{3} \mathrm{~T} 31$ \\
\hline 53 & $5: 38: 30$ & $-02: 40: 10$ & $0 \cdot 7 \times 0 \cdot 6$ & b & \\
\hline 54 & $5: 38: 31$ & $-02: 47: 20$ & $0 \cdot 9 \times 0 \cdot 4$ & b & \\
\hline 55 & $5: 38: 42$ & $-06: 37: 00$ & $0 \cdot 6 \times 1 \cdot 5$ & c & \\
\hline 56 & 5:39:11 & $-06: 28: 10$ & $1 \cdot 7 \times 14 \cdot 5$ & c & \\
\hline $57 \mathrm{~A}$ & $5: 39: 27$ & $-03: 04: 30$ & $2 \cdot 7 \times 3 \cdot 4$ & b & \\
\hline $57 \mathrm{~B}$ & $5: 39: 25$ & $-03: 02: 20$ & $2 \cdot 0 \times 1 \cdot 3$ & $\mathrm{~b}$ & \\
\hline 58 & 5:39:31 & $-02: 59: 30$ & $2 \cdot 2 \times 1 \cdot 5$ & $\mathrm{~b}$ & \\
\hline 59 & $5: 40: 40$ & $-05: 21: 40$ & $2 \cdot 1 \times 6 \cdot 7$ & c & \\
\hline $60 \mathrm{~A}$ & $5: 40: 54$ & $-05: 02: 20$ & $4 \cdot 1 \times 14 \cdot 5$ & c & \\
\hline $60 \mathrm{~B}$ & $5: 41: 24$ & $-05: 06: 50$ & $1 \cdot 3 \times 6 \cdot 7$ & $\mathrm{c}$ & \\
\hline $61 \mathrm{~A}$ & 5:40:59 & $-03: 36: 50$ & $5 \cdot 0 \times 5 \cdot 0$ & $\mathrm{c}$ & \\
\hline 61B & $5: 41: 02$ & $-03: 30: 00$ & $2 \cdot 7 \times 3 \cdot 9$ & c & \\
\hline 62 & $5: 44: 28$ & 00:38:10 & $3 \cdot 1 \times 4 \cdot 0$ & c & DG82? \\
\hline CC-A tip & 5:04:50 & $-06: 20: 00$ & $40 \times 350$ & c & IC 2118, LBN959, DG52 \\
\hline $\mathrm{CC}-\mathrm{A}$ end & 4:54:00 & $-11: 30: 00$ & & & \\
\hline
\end{tabular}

* BRC: Sugitani et al. (1991); CB: Clemens \& Barvainis (1988); $\mathrm{M}^{3} \mathrm{~T}$ : Maddalena et al. (1986); LBN: Lynds (1965); DG: Dorschner \& Gürtler (1964).

Cometary globules generally have brighter outlines near the apexes than at the rest of their boundaries, whereas reflection clouds are more or less of uniform brightness over their boundaries and interiors. Still the discrimination between cometary globules and reflection clouds based only on Schmidt atlases is sometimes difficult and requires spectroscopic data on the rims. These criteria are admittedly subjective.

Table 1 summarises the results. The first column gives the object number identified in Figure 1. The second and third columns contain the 1950 coordinates of the cloud centre. Since most of the objects are more or less elongated with a sparse tail, the coordinates refer to the centres of their heads. The fourth and fifth columns give the apparent size and the cloud type, respectively. It is apparent from Figure 1 that remnant clouds of the three categories are essentially similar in size. The mean size of all the objects is $2 \cdot 6^{\prime} \times 4 \cdot 9^{\prime}$ or $0 \cdot 35 \mathrm{pc} \times 0 \cdot 66 \mathrm{pc}$ at the adopted distance of $460 \mathrm{pc}$ with the range of $\left(0 \cdot 4-14 \cdot 0^{\prime}\right) \times\left(0 \cdot 4-33 \cdot 6^{\prime}\right)$ or $(0 \cdot 05-1 \cdot 87 \mathrm{pc}) \times(0 \cdot 05-$ $4 \cdot 49 \mathrm{pc})$. Identifications from previous catalogues are found in the last column. In addition to these objects one notices a huge structure of roughly cometary shape in the far south-western region. It stretches about $5 \cdot 8^{\circ}$ with a width of roughly $40^{\prime}$. We name it Cometary Complex (CC)-A.

\section{Discussion}

In Figure 1 one sees that not only bright-rimmed clouds and cometary globules but also most reflection clouds have elongated tails. Figure 2 shows the surface distribution of all the objects in Table 1 together with the directions of their tails. The tails of nearby clouds are apparently aligned in a common direction. More interestingly all the clouds as a whole point roughly to a common centre, which is located somewhere in the Belt or Sword region. (As for the huge $\mathrm{CC}-\mathrm{A}$, the extension of its axis passes far north, but the bright part of its jagged outline appears to point to the Belt region.) The same trend was also found by Bally et al. (1991) in their $\mathrm{CO}(J=1-0)$ and ${ }^{13} \mathrm{CO}(J=1-0)$ survey in the outer Orion region. This suggests that these structures have been formed by a common action, which is most probably the UV radiation from the past and/or present $\mathrm{O}$ stars in the Ori OB1 Association. In particular for many clouds in the Belt region the shaping centre is the O7V star $\sigma$ Ori, which is the exciting star of IC 434.

In the Belt region one finds a spatial sequence of the three categories of remnant clouds. Brightrimmed clouds including the Horsehead Nebula (object no. 52) are located closest to $\sigma$ Ori. To the north-west of the star there are several cometary globules, among which Ori I-2 (object no. 40A) is the most prominent. Further to the north-west a group of reflection clouds (typified by object no. 27) are found. This beautiful arrangement strongly suggests the evolutionary sequence of remnant clouds starting from bright-rimmed clouds through cometary globules to reflection clouds. However, remnant clouds which are located far from the $\mathrm{O}$ star(s) may directly become cometary globules or reflection clouds. 

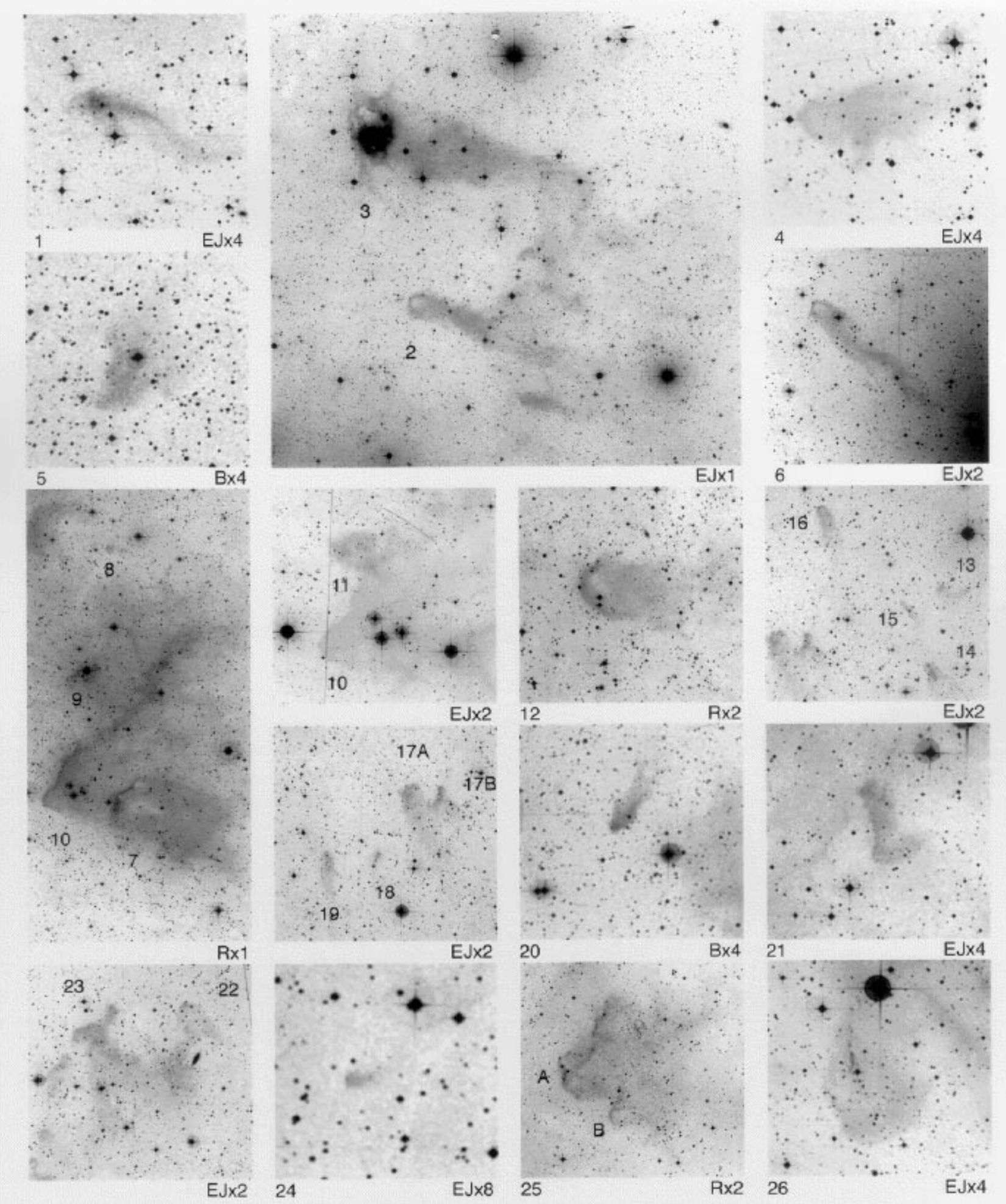

Figure 1-Identification charts for the objects listed in Table 1. These are reproduced from the SERC Equatorial EJ Sky Atlas (indicated by EJ), the Second Palomar Sky Survey B Atlas (indicated by B) or red plates taken with the UK Schmidt telescope (indicated by R). The magnification follows the colour band. North is up and east to the left. 

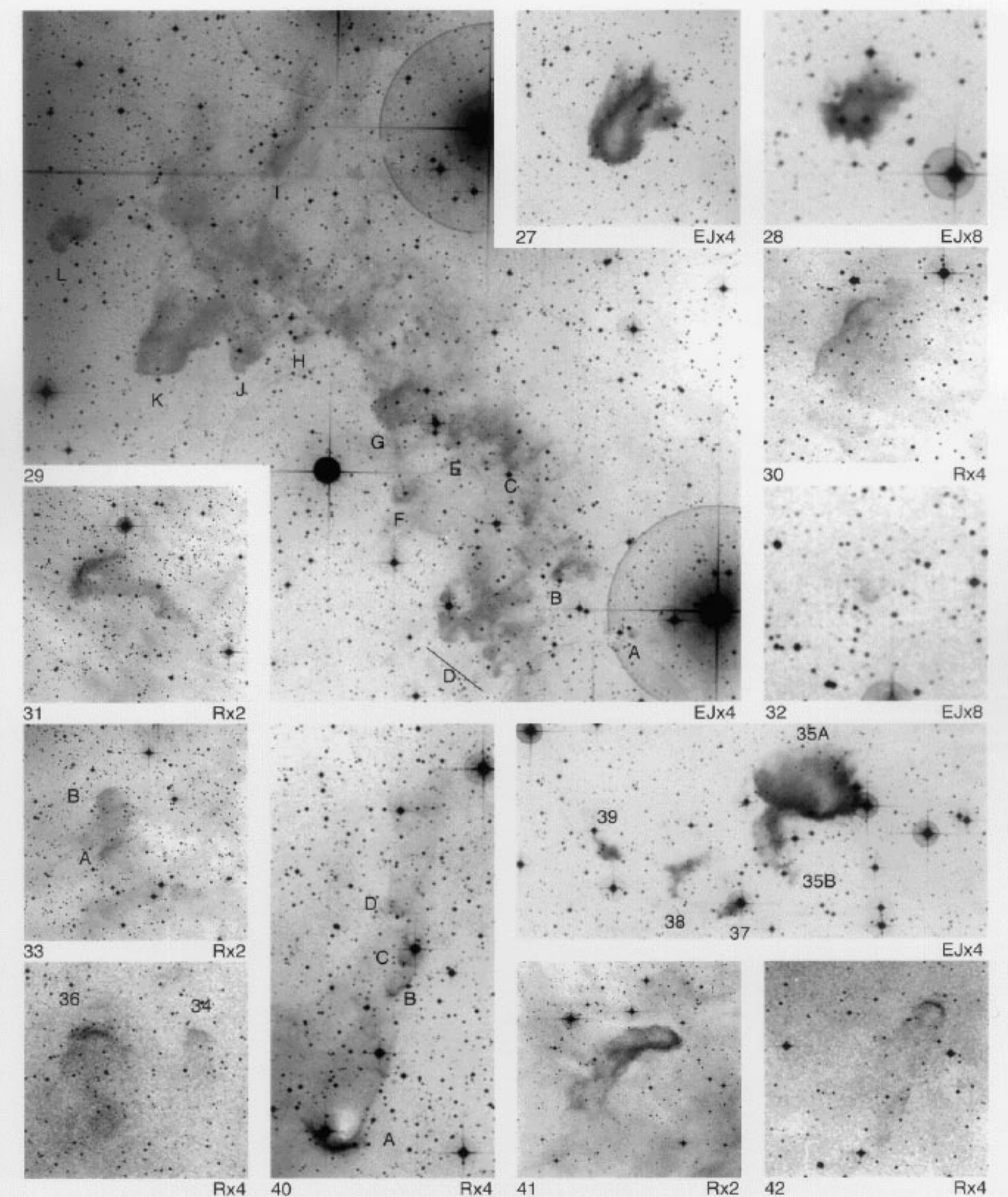

Figure 1-Continued. 

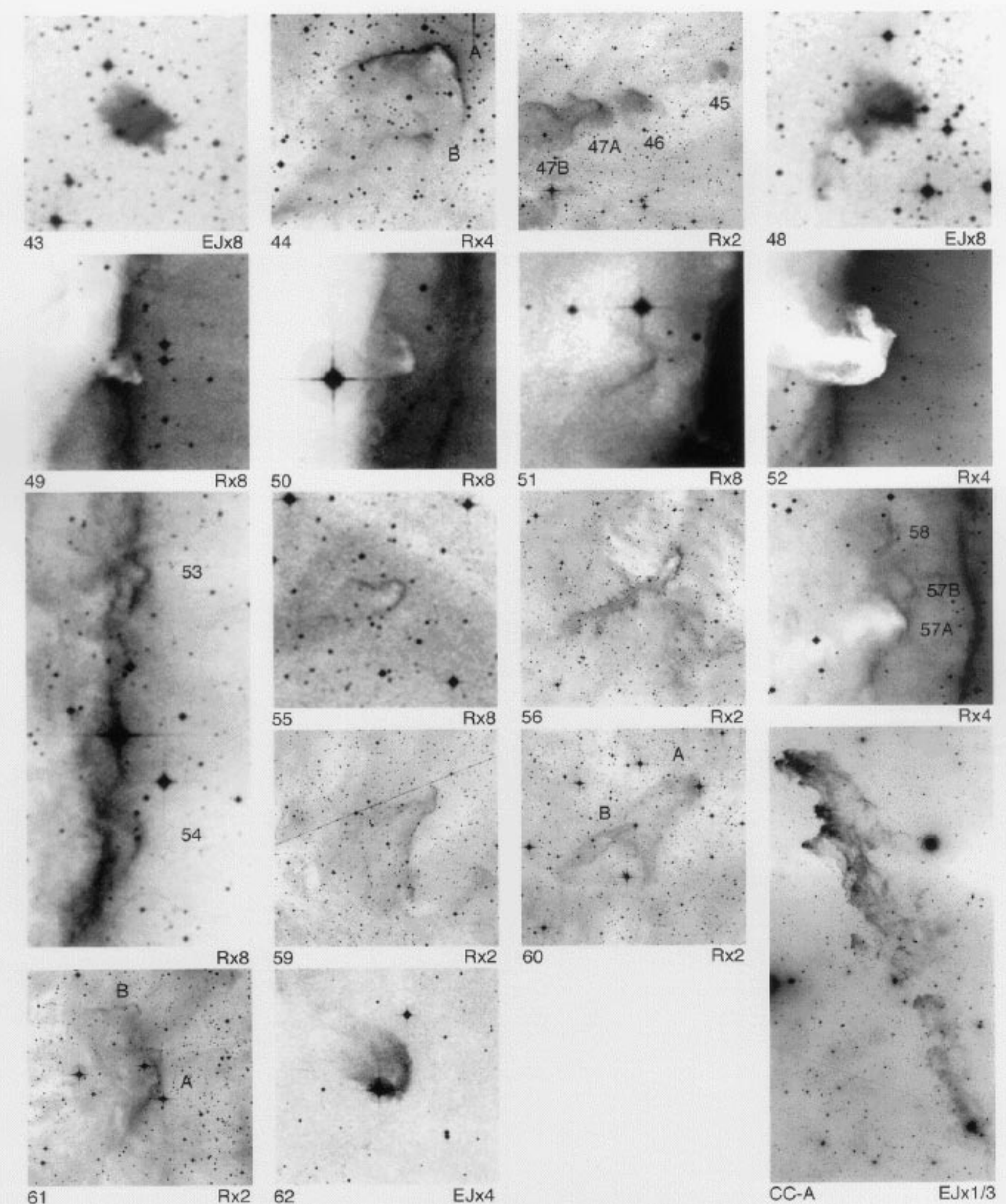

Figure 1-Continued. 


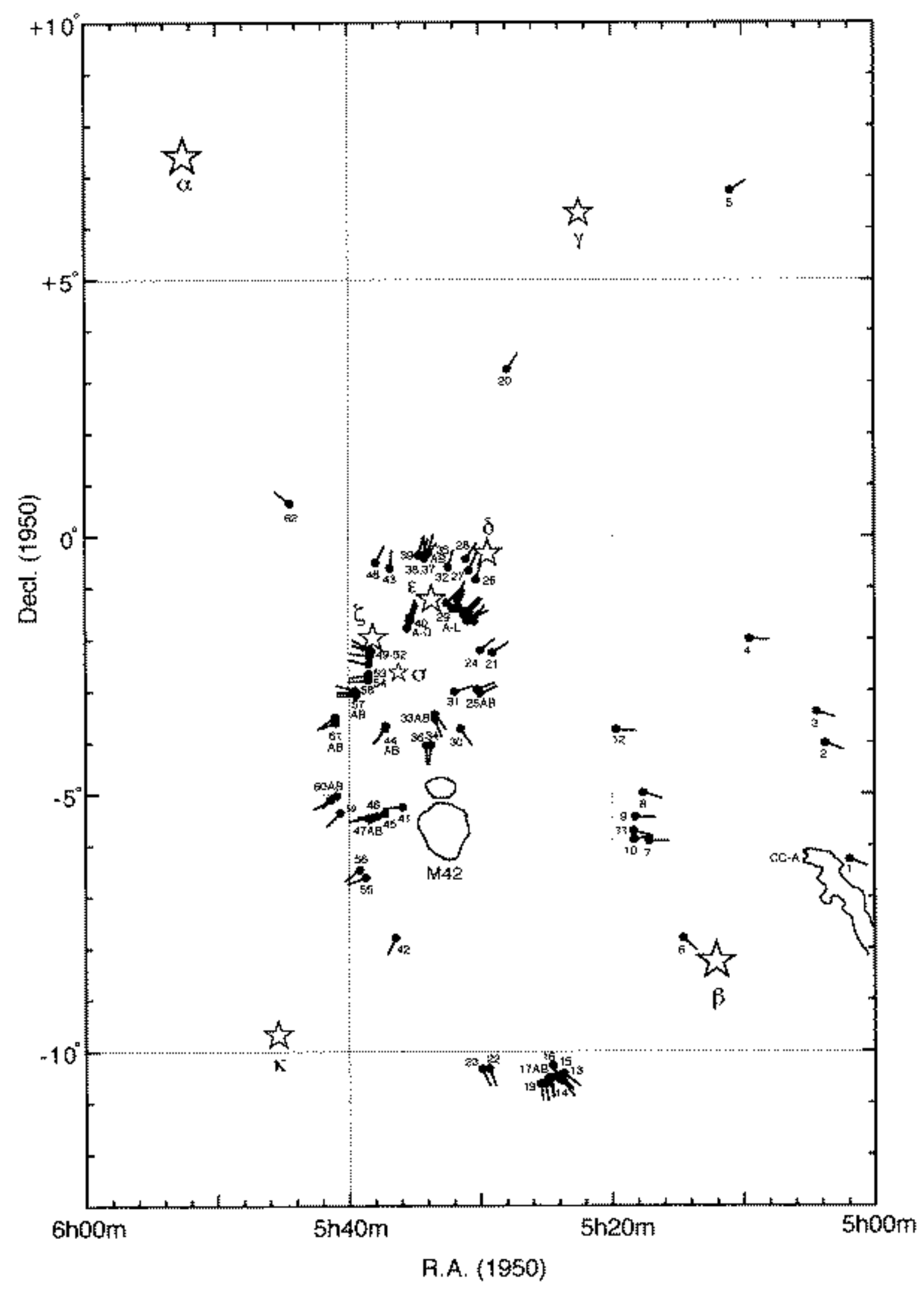

Figure 2-Surface distribution of the objects in Table 1. Ticks indicate the directions of their tails. 
As already mentioned there is plenty of evidence for recent or ongoing star formation in bright-rimmed clouds and cometary globules. For selected reflection clouds in the Belt region we have carried out $\mathrm{CO}$ $(J=1-0),{ }^{13} \mathrm{CO} \quad(J=1-0)$ and $\mathrm{C}^{18} \mathrm{O} \quad(J=1-0)$ observations as well as IR imaging. The molecular nature of the clouds has been confirmed and young stellar objects have been found associated with some of them. These results will be reported in a forthcoming paper (Nakano et al. 1998).

One of the foci in recent studies of star formation is the so-called dispersed $\mathrm{T}$ Tauri stars, which are found far outside molecular clouds. Three explanations have so far been proposed as to their origin or nature: runaway $\mathrm{T}$ Tau stars (Sterzik \& Durisen 1995), star formation in high-velocity cloudlets (Feigelson 1996) and young main-sequence field stars (Briceño et al. 1997). In the Orion region Sterzik et al. (1995) identified many such candidates by using the ROSAT All Sky Survey. The objectiveprism Schmidt surveys of our group (Nakano, Kogure \& Wiramihardja 1995 and references therein) have also detected altogether over $1000 \mathrm{H} \alpha$ emission stars in a similar sky area to that of the present study. We suspect that retarded star formation in remnant clouds can explain the presence of dispersed $\mathrm{T}$ Tauri stars at least in the peripheries of Orion OB 1 and other OB associations.
Bally, J., Langer, W. D., Wilson, R. W., Stark, A. A., \& Pound, M. W. 1991, in IAU Symp. 147, Fragmentation of Molecular Clouds and Star Formation, ed. I. Falgarone, F. Boulanger \& G. Duvert (Dordrecht: Kluwer), p. 11

Briceño, C., Hartmann, L. W., Stauffer, J. R., Gagne, M., Stern, R. A., \& Caillault, J.-P. 1997, AJ, 113, 740

Clemens, D. P., \& Barvainis, R. 1988, ApJS, 68, 257

Dorschner, J., \& Gürtler, J. 1964, Astron. Nachr., 287, 257

Feigelson, E. D. 1996, ApJ, 468, 306

Lefloch, B., \& Lazareff, B. 1994, A\&A, 289, 559

Maddalena, R. J., Morris, M., Moscowitz, J., \& Thaddeus, P. 1986, ApJ, 303, 375

Lynds, B. T. 1965, ApJS, 12, 163

Maddalena, R. J., Morris, M., Moscowitz, J., \& Thaddeus, P. 1986, ApJ, 303, 375

Nakano, M., Kogure, T., \& Wiramihardja, S. 1995, PASJ, 48,889

Nakano, M., Ogura, K., Sugitani, K., \& Tamura, M. 1998, in preparation

Reipurth, B. 1983, A\&A, 117, 183

Sterzik, M. F., Alcala, J. M., Neuhäuser, R., \& Schmidt, J. H. M. M. 1995, A\&A, 297, 418

Sterzik, M. F., \& Durisen, R. H. 1995, A\&A, 304, L9

Sugitani, K., Fukui, Y., \& Ogura, K. 1991, ApJS, 77, 59

Sugitani, K., \& Ogura, K. 1994, ApJS, 92, 163

Sugitani, K., Tamura, M., \& Ogura, K. 1995, ApJL, 455, L39

Tatematsu, K., et al. 1993, ApJ, 404, 643

Zealey, W. J., Ninkov, Z., Rice, E., Hartley, M., \& Tritton, S. B. 1983, ApJ Lett., 23, 119 